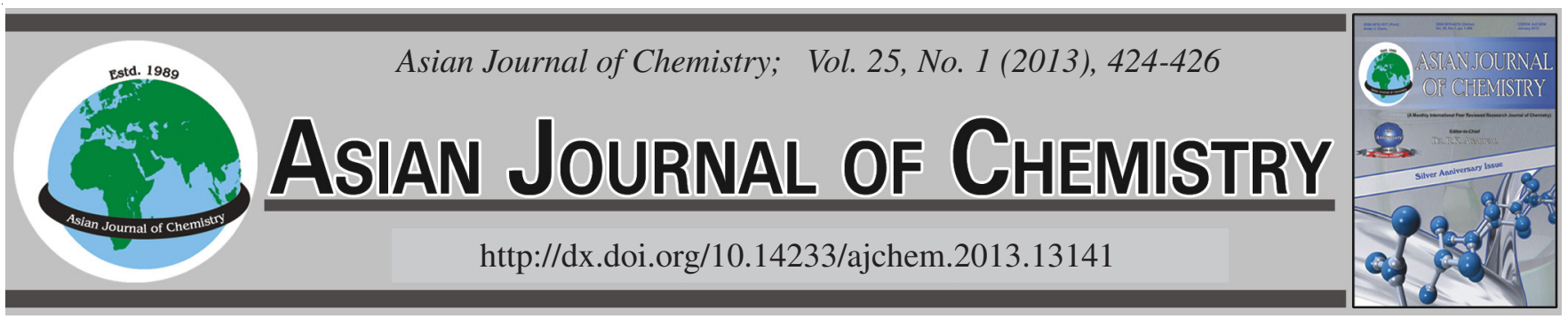

\title{
Phenolic Compound Content in Different Organs of Korean Common Buckwheat Cultivars
}

\author{
Md Romis Uddin ${ }^{1}$, XiaOhua Li $^{1}$, Woo Tae Park ${ }^{1}$, Yeon Bok Kim ${ }^{1}$, Sun Ju Kim ${ }^{2}$, \\ Young Seon Kim ${ }^{3}$, Mi Young LeE ${ }^{3}$, Cheol Ho Park and Sang Un Park ${ }^{1, *}$
}

\begin{abstract}
${ }^{1}$ Department of Crop Science, College of Agriculture \& Life Sciences, Chungnam National University, 79 Daehangno, Yuseong-gu, Daejeon, 305-764, South Korea

${ }^{2}$ Department of Bio-Environmental Chemistry, College of Agriculture \& Life Sciences, Chungnam National University, 99 Daehangno, Yuseong-gu, Daejeon, 305-764, South Korea

${ }^{3}$ TKM-Based Herbal Drug Research Group, Korea Institute of Oriental Medicine, Daejeon, 305-811, South Korea

${ }^{4}$ Department of Bio-Health Technology, College of Biomedical Science, Kangwon National University, 192-1 Hyoja 2-Dong, Chuncheon 200-701, South Korea
\end{abstract}

*Corresponding author: Fax: +82 42 8222631; Tel: +82 42 8215730; E-mail: supark@cnu.ac.kr

\section{INTRODUCTION}

Fagopyrum esculentum Moench (common buckwheat) and Fagopyrum tataricum Gaertn (tartary buckwheat) are important members of the genus, Fagopyrum that are used for food around the world, common buckwheat originated from southwest China and has gradually spread to every continent, whereas tartary buckwheat is grown and used in the mountainous regions of southwest China (Sichuan), northern India, Bhutan and Nepal and only in a small part of northwest Europe $^{1,2}$.

In recent years, dietary plants such as buckwheat have attracted attention because they contain antioxidants that protect the human body from oxidative damage caused by free radicals. Buckwheat grain has a higher antioxidative activity than other cereal grains ${ }^{3}$; its antioxidative compounds include vitamins such as vitamins B1, B2 and E, as well as several phenolic compounds, which are found in the organs of buckwheat (leaf, stem and inflorescence) such as rutin, quercetin and proanthocyanidines (condensed tannins) ${ }^{4-6}$.
Phenolic compounds in foods belong to one of the main classes of secondary metabolites and are widely distributed in higher plants. Currently, research interest in these substances has been stimulated by the potential health benefits arising from the antioxidant activity of these polyphenolic compounds, which protect the body's tissues against and the pathologies associated with oxidative stress, such as cancers, coronary heart disease and inflammation ${ }^{7-10}$.

The objective of this study was to determine the levels of phenolic compounds in different organs and across various cultivars, of common buckwheat.

\section{EXPERIMENTAL}

F. esculentum seeds were donated by the National Agrobiodiversity Center, National Academy of Agricultural Science, Rural Development Administration, Korea and stored at $4{ }^{\circ} \mathrm{C}$. F. esculentum seeds were germinated in a greenhouse and the seedlings were transferred to the experimental farm at Chungnam National University, Daejeon, Korea. After 3 months, the roots, stems, leaves and flowers of different 
cultivars of $F$. esculentum were collected for chemical analysis of phenolic compounds.

HPLC analysis of phenolic compounds: The fresh organ samples collected from the different $F$. esculentum cultivars were stored in sealed clear polyethylene plastic bags at $-80^{\circ} \mathrm{C}$ until they were used. Samples were dried in a freeze-dryer at $-80{ }^{\circ} \mathrm{C}$ for at least $48 \mathrm{~h}$, after which the dried samples were ground into a fine powder by using a mortar and pestle. Dried and ground samples $(0.1 \mathrm{~g})$ were extracted with $3 \mathrm{~mL}$ pure methanol at $60{ }^{\circ} \mathrm{C}$ for $0.5 \mathrm{~h}$ to obtain rutin and quercetin and extracted twice with $3 \mathrm{~mL}$ of $80 \%$ methanol for $1 \mathrm{~h}$, at room temperature, to obtain the remaining test compounds. The extract solutions were filtered through a $0.45 \mu \mathrm{m}$ poly filter and then diluted two-fold with methanol prior to high performance liquid chromatography (HPLC) analysis.

HPLC quantification of phenolic compounds was performed using a Futecs model NS-4000 HPLC apparatus (Daejeon, Korea). The analysis was monitored at $280 \mathrm{~nm}$ and performed using a C18 column $(250 \mathrm{~mm} \times 4.6 \mathrm{~mm}, 5 \mu \mathrm{m}$; RStech, Daejeon, Korea). A gradient, prepared from mixtures of acetonitrile and $0.15 \%$ acetic acid, was used as the mobile phase and the column was maintained at $30^{\circ} \mathrm{C}$. The flow rate was set at $1.0 \mathrm{~mL} / \mathrm{min}$ and the injection volume was $20 \mu \mathrm{L}$. The resulting concentrations of phenolic compounds were calculated using a standard curve. All samples were run in triplicates.

\section{RESULTS AND DISCUSSION}

Phenolic compounds in the leaf: During the analysis of common buckwheat leaf, the presence of seven different phenolic compounds was recorded. Among these, the levels of rutin were much higher than that of any other compound (Table-1). The rutin content in 3 common buckwheat cultivars ranged from 7.23 to $13.43 \mu \mathrm{g} / \mathrm{mg}$ dry weight, representing a 1.9-fold difference between the Suwon 12 and Suwon 1 cultivars, which had the highest and lowest rutin content, respectively. The content of epicatechin ranged from 0.34 to 0.12 $\mu \mathrm{g} / \mathrm{mg}$ dry weight, among the studied cultivars; the cultivar Suwon 1 contained the highest quantity of epicatechin, measuring 2.83- fold higher than the lowest content cultivar, Suwon 2 (Table-1). The amounts of other compounds such as 4-hydroxy benzoic acid, catechin, 4-hydroxy-3-methoxy benzoic acid and $p$-coumaric acid were lower in the leaf parts of all the cultivars.than in other parts of the plants (Table-1).

Phenolic compounds in the stem: All the seven phenolic compounds mentioned above were also identified during the analysis of the stem of common buckwheat (Table-1). It was found that the 4-hydroxy benzoic acid, catechin, chlorogenic acid and $p$-coumaric acid contents were higher, whereas, the rutin content was lower in the stem than in the leaf, while the others compounds did not vary remarkably between the leaf and stem. The trends in phenolic compound content among the cultivars were similar in stem and leaf, but the variation was slightly higher for stems than for leaves. The rutin content in different common buckwheat cultivars ranged from 2.38 to $4.78 \mu \mathrm{g} / \mathrm{mg}$ dry weight. This represents a two-fold difference between Suwon 2 and Suwon 1 cultivars, which had the highest and lowest rutin content, respectively. The content of catechin and 4-hydroxy benzoic acid ranged from 0.04 to 0.26 and 0.05 to $0.18 \mu \mathrm{g} / \mathrm{mg}$ dry weight, respectively, among the studied cultivars. The cultivar Suwon 2 contained the highest levels of catechin and 4-hydroxy benzoic acid, which were 6.5-fold and 3.6-fold, respectively, higher than the lowest content cultivar Suwon 12.

Phenolic compounds in the flower: In flowers, we identified 8 phenolic compounds, including quercetin and kaemferol, which were not present in leaves and stem, but excluding 4-hydroxy benzoic acid, which had been found in the leaves and stems (Table-1) were during the analysis of common buckwheat flower. A markedly higher amount of catechin, chlorogenic acid, epicatechin, rutin and quercetin were observed in the flower than in other parts of the plant (Table-1). The content of catechin in the flower ranged from 0.55 to $2.22 \mu \mathrm{g} / \mathrm{mg}$ dry weight among the studied cultivars. In the highest catechincontent cultivar Suwon 1, the catechin level in the flower was 4.0-fold higher than in the lowest content cultivar Suwon 2 and 74-fold and 55.5-fold higher than that in the leaf and stem, respectively, of the lowest catechin content cultivars (Table-1). The content of chlorogenic acid in the flower was also vastly higher than that in the leaf and stem. The range of chlorogenic acid content in the flower of common buckwheat cultivars was 4.93 to $10.54 \mu \mathrm{g} / \mathrm{mg}$ dry weight (Table-1). The Suwon 2 cultivar contained the highest amount of chlorogenic acid,

\begin{tabular}{|c|c|c|c|c|c|c|c|c|c|c|}
\hline \multirow{3}{*}{ Organs } & \multicolumn{10}{|c|}{$\begin{array}{l}\text { TABLE-1 } \\
\text { VARIATION OF PHENOLIC COMPOUNDS IN DIFFERENT PARTS (LEAF, STEM, } \\
\text { FLOWER AND ROOT) OF KOREAN COMMON BUCKWHEAT CULTIVARS }\end{array}$} \\
\hline & \multirow{2}{*}{ Cultivars } & \multicolumn{9}{|c|}{ Phenolic compounds ( $\mu \mathrm{g} / \mathrm{mg}$ DW) } \\
\hline & & I & II & III & IV & $\mathrm{V}$ & VI & VII & VIII & IX \\
\hline \multirow{3}{*}{ Flower } & Suwon 1 & $0.00 \pm 0.00$ & $2.22 \pm 0.11$ & $4.93 \pm 0.04$ & $0.67 \pm 0.05$ & $3.18 \pm 0.16$ & $0.04 \pm 0.01$ & $31.58 \pm 2.10$ & $0.18 \pm 0.03$ & $0.00 \pm 0.00$ \\
\hline & Suwon 2 & $0.00 \pm 0.00$ & $0.55 \pm 0.09$ & $10.54 \pm 1.2$ & $0.83 \pm 0.03$ & $3.82 \pm 0.21$ & $0.03 \pm 0.01$ & $56.10 \pm 1.18$ & $0.32 \pm 0.06$ & $0.00 \pm 0.00$ \\
\hline & Suwon 12 & $0.00 \pm 0.00$ & $1.04 \pm 0.03$ & $5.93 \pm 0.19$ & $0.79 \pm 0.03$ & $1.83 \pm 0.14$ & $0.04 \pm 0.00$ & $44.31 \pm 2.05$ & $0.19 \pm 0.03$ & $0.02 \pm 0.00$ \\
\hline \multirow{3}{*}{ Leaf } & Suwon 1 & $0.11 \pm 0.00$ & $0.06 \pm 0.00$ & $0.13 \pm 0.00$ & $0.05 \pm 0.00$ & $0.34 \pm 0.00$ & $0.01 \pm 0.00$ & $7.23 \pm 0.03$ & $0.00 \pm 0.00$ & $0.00 \pm 0.00$ \\
\hline & Suwon 2 & $0.14 \pm 0.00$ & $0.03 \pm 0.00$ & $0.20 \pm 0.00$ & $0.02 \pm 0.00$ & $0.12 \pm 0.00$ & $0.00 \pm 0.00$ & $9.76 \pm 0.13$ & $0.00 \pm 0.00$ & $0.00 \pm 0.00$ \\
\hline & Suwon 12 & $0.06 \pm 0.00$ & $0.04 \pm 0.00$ & $0.16 \pm 0.01$ & $0.07 \pm 0.01$ & $0.15 \pm 0.02$ & $0.01 \pm 0.00$ & $13.43 \pm 0.53$ & $0.00 \pm 0.00$ & $0.00 \pm 0.00$ \\
\hline \multirow{3}{*}{ Stem } & Suwon 1 & $0.09 \pm 0.00$ & $0.09 \pm 0.01$ & $0.30 \pm 0.01$ & $0.04 \pm 0.00$ & $0.20 \pm 0.00$ & $0.07 \pm 0.01$ & $2.38 \pm 0.17$ & $0.00 \pm 0.00$ & $0.00 \pm 0.00$ \\
\hline & Suwon 2 & $0.18 \pm 0.00$ & $0.26 \pm 0.01$ & $0.50 \pm 0.01$ & $0.03 \pm 0.00$ & $0.31 \pm 0.01$ & $0.10 \pm 0.01$ & $4.78 \pm 0.03$ & $0.00 \pm 0.00$ & $0.00 \pm 0.00$ \\
\hline & Suwon 12 & $0.05 \pm 0.00$ & $0.04 \pm 0.00$ & $0.38 \pm 0.01$ & $0.06 \pm 0.00$ & $0.14 \pm 0.02$ & $0.03 \pm 0.00$ & $2.81 \pm 0.04$ & $0.00 \pm 0.00$ & $0.00 \pm 0.00$ \\
\hline \multirow{3}{*}{ Root } & Suwon 1 & $0.00 \pm 0.00$ & $0.12 \pm 0.00$ & $0.07 \pm 0.00$ & $0.09 \pm 0.00$ & $0.40 \pm 0.00$ & $0.10 \pm 0.00$ & $0.08 \pm 0.01$ & $0.00 \pm 0.00$ & $0.02 \pm 0.00$ \\
\hline & Suwon 2 & $0.00 \pm 0.00$ & $0.18 \pm 0.02$ & $0.09 \pm 0.00$ & $0.04 \pm 0.00$ & $0.20 \pm 0.01$ & $0.12 \pm 0.00$ & $0.20 \pm 0.04$ & $0.00 \pm 0.00$ & $0.01 \pm 0.00$ \\
\hline & Suwon 12 & $0.00 \pm 0.00$ & $0.09 \pm 0.01$ & $0.07 \pm 0.00$ & $0.06 \pm 0.00$ & $0.25 \pm 0.01$ & $0.09 \pm 0.01$ & $0.19 \pm 0.01$ & $0.00 \pm 0.00$ & $0.03 \pm 0.00$ \\
\hline
\end{tabular}

$\mathrm{I}=4$-Hydroxy benzoic acid; II = Catechin; III = Chlorogenic acid; IV = 4-Hydroxy-3-methoxy benzoic acid; V = Epicatechin; VI = p-Coumaric acid; VII = Rutin; VIII = Quercetin; IX = Kaempferol. 
which was 2.1-fold higher than that of the lowest content cultivar Suwon 1. The content of chlorogenic acid in the flower was 81.1-fold and 35.1-fold higher than that in the leaf and stem, respectively, of the lowest chlorogenic acid content cultivars. The epicatechin content in different common buckwheat cultivars in the flower ranged from 1.83 to $3.82 \mu \mathrm{g} / \mathrm{mg}$ dry weight (Table-1), representing a 2.1-fold difference between the Suwon 2 and Suwon 12 cultivars, which had the highest and lowest epicatechin content, respectively. The epicatechin content in flower was 31.8-fold and 27.3-fold higher than that in the leaf and stem, respectively, of the lowest epicatechin content-cultivars. The rutin content in the flowers of different common buckwheat cultivars ranged from 31.58 to $56.1 \mu \mathrm{g} / \mathrm{mg}$ dry weight (Table-1). This represents a 1.8 -fold difference between Suwon 2 and Suwon 1 cultivars, which had the highest and lowest rutin content, respectively. The rutin content in the flower was 7.8-fold and 23.6-fold higher than that in the leaf and stem, respectively, of the lowest rutin content cultivars. The range of quercetin content in the flower of common buckwheat cultivars ranged from 0.18 to $0.32 \mu \mathrm{g} / \mathrm{mg}$ dry weight (Table-1). Quercetin was absent both in leaf and stem parts of common buckwheat. The Suwon 2 cultivar contained the highest amount of quercetin, which was 1.8-fold higher than the lowest content cultivar Suwon 1. The content of 4-hydroxy-3methoxy benzoic acid in the flower was much higher than that in the leaf and stem of common buckwheat cultivars (Table1). Suwon 2 contained the highest amount of 4-hydroxy-3methoxy benzoic acid, while Suwon 1 contained the lowest amount of 4-hydroxy-3-methoxy benzoic acid. The 4-hydroxy3-methoxy benzoic content in the flower was 41.5- and 27.7fold higher than that in the leaf and stem, respectively, of the lowest 4-hydroxy-3-methoxy benzoic content cultivars.

Phenolic compounds in the root: The level of phenolic compounds was much lower in the root of common buckwheat compared to the leaf, stem and flowers (Table-1). Seven phenolic compounds were observed in the root; these did not include 4-hydroxy benzoic acid and or quercetin. The amount of epicatechin was higher than that of other phenolic compounds in the root. The epicatechin content in different common buckwheat cultivars in the root ranged from 0.20 to $0.40 \mu \mathrm{g} /$ mg dry weight. This represents a 2.0 -fold difference between Suwon 1 and Suwon 2 cultivars, which had the highest and lowest epicatechin content, respectively.

The results of this study show that the flower of common buckwheat contained far more phenolic compounds than the other plant parts. It is also found that the cultivars had a great influence on the content of phenolic compounds; some cultivars contained more phenolic compounds, whereas some others contained less. It is notable that the trend for phenolic compound levels in the different flower parts were not always the same in a given cultivar. For example, the Suwon 2 cultivar contained the highest amount of rutin of all tested cultivars in the flower, stem and root, but in the leaf of Suwon 2, the amount of rutin was not the highest among these cultivars.

There have been some previous reports of varietal differences in the antioxidative components in buckwheat, including differences in the rutin content ${ }^{11,12}$, while varietal and environmental differences in vitamin $\mathrm{E}^{13}$ and phenolic acid $^{14}$ have also been described. Moreover, the antioxidative activity of ethanol extract from buckwheat seeds has been reported to vary according to the cultivar and correlated with the polyphenol content ${ }^{3}$.

Flavonoid content has also been suggested to be dependent on the cultivar ${ }^{15}$, as large variation in the flavonoid content among the cultivars of flaxseed (Linum usitatissimum) has previously been described ${ }^{16}$. Furthermore, flavonoid content and antioxidant activity in almonds may be more dependent on the cultivar than on seasonal differences ${ }^{17}$. A study of 10 Portuguese almond cultivars found 4-fold and 18-fold differences in the content of flavonoids and total phenolic compounds, respectively, between cultivars ${ }^{18}$. Moreover, analysis of 18 Iranian almond genotypes showed a 4.6-fold variation in the phenolic content of hulls and a similar variability in the antioxidant activity of extracts ${ }^{19}$. In present study, similar results were observed in the variation of phenolic compound content in the cultivars of common buckwheat.

\section{Conclusion}

The levels of phenolic compounds in different parts of common buckwheat vary widely. The flowers were found to have the highest levels of all the phenolic compounds identified during the analysis of the plant organ samples. The type of cultivar also greatly influenced the content of phenolic compounds in the plant parts. Cultivar-specific phenolic compound profiles might be helpful for commercial purposes or may facilitate planned production of phenolic compounds.

\section{ACKNOWLEDGEMENTS}

This study was supported by Technology Development Program for Agriculture and Forestry, Ministry for Food, Agriculture, Forestry and Fisheries, Republic of Korea.

\section{REFERENCES}

1. G. Bonafaccia, L. Gambelli, N. Fabjan and I. Kreft, Food Chem., 83, 1 (2003).

2. T.D. Xuan and E. Tsuzuki, Allelopathy J., 13, 137 (2004).

3. H. Zielinski and H. Kozlowska, J. Agric. Food Chem., 48, 2008 (2000).

4. M. Watanabe, A. Sato, R. Ohsawa and J. Terao, Nippon Shokuhin Kogyo Gakkaishi (Japan), 42, 649 (1995).

5. M. Watanabe, Y. Ohshi and T. Tsushida, J. Agric. Food Chem., 45, 1039 (1997).

6. M. Watanabe, J. Agric. Food Chem., 46, 839 (1998)

7. K.D. Croft, Ann. NY Acad. Sci., 854, 435 (1998).

8. S. Karakaya, Crit. Rev. Food Sci. Nutr., 44, 453 (2004).

9. J. Linseisen and S. Rohrmann, Eur. J. Nutr., 47, 60 (2008).

10. H. Tapiero, K.D. Tew, G.N. Ba and G. Mathé, Biomed. Pharmacother, 56, 200 (2000).

11. H. Kitabayashi, A. Ujihara, T. Hirose and M. Minami, Breed Sci., 45, 189 (1995).

12. R. Ohsawa and T. Tsutsumi, Euphytica, 86, 183 (1995).

13. Y. Honda, Proc. 6th Intl. Symp. Buckwheat at Ina (1995).

14. B.D. Oomah, C.G. Campbell and G. Mazza, Euphytica, 90, 73 (1996).

15. P.E. Milbury, C.Y. Chen, G.G. Dolnikowski and J.B. Blumberg, J. Agric. Food Chem., 54, 5027 (2006).

16. B.D. Oomah, G. Mazza and E.O. Kenaschuk, Euphytica, 90, 163 (1996).

17. B.W. Bolling, G. Dolnikowski, J.B. Blumberg and C.Y.O. Chen, Food Chem., 122, 819 (2010).

18. J.C. Barreira, I.C. Ferreira, M.B. Oliveira and J.A. Pereira, Food Chem. Toxicol., 46, 2230 (2008).

19. A.J. Sfahlan, A. Mahmoodzadeh, A. Hasanzadeh, R. Heidari and R. Jamei, Food Chem., 115, 529 (2009). 